\title{
AVALIAÇÃO DA VULNERABILIDADE NATURAL DO SOLO EM ÁREAS AGRÍCOLAS: SUBSÍDIO À AVALIAÇÃO DO RISCO DE CONTAMINAÇÃO DO LENÇOL FREÁTICO POR AGROQUÍMICOS
}

\author{
MARCO ANTONIO FERREIRA GOMES * \\ CLÁUDIO A. SPADOTTO ** \\ MARIA CONCEIÇÃO P. Y. PESSOA***
}

\begin{abstract}
Procurou-se mostrar que a vulnerabilidade natural do solo é um parâmetro fundamental nos estudos de avaliação de riscos ambientais, sobretudo em áreas de grande fragilidade, como as áreas de recarga dos aqüíferos sedimentares. Para esse estudo foi adotada uma área típica de recarga direta do Aqüífero Guarani, caracterizada pela microbacia do Córrego do Espraiado, localizada na região de Ribeirão Preto/SP (Brasil). Os procedimentos consistiram na integração dos parâmetros condutividade hidráulica, declividade e profundidade do lençol freático dos principais solos da área objeto de estudo. A condutividade hidráulica foi determinada com a adoção do método da coluna saturada, com valores considerados a partir de estimativa classificada em baixa, média e alta. Nessa estimativa, levou-se em conta a integração dos dados de textura, estrutura, estabilidade de agregados e profundidade do solo, que foram submetidos a tratamento estatístico para validação do método proposto. As correlações foram diretas entre condutividade hidráulica medida e condutividade hidráulica estimada. A declividade da área foi obtida a partir de mapas planialtimétricos, em escala 1:10.000, que permitiram o agrupamento em três classes: baixa, suave e acentuada. Seqüencialmente, foi estabelecida relação matricial entre as classes de condutividade hidráulica e as de declividade, resultando na classificação relativa dos potenciais de infiltração e de escoamento superficial da água para toda a microbacia. Concluiu-se que essas informações combinadas com aquelas relativas à condutividade hidráulica, declividade do terreno e profundidade do solo permitiram, de forma satisfatória, estimar em três classes (baixa, média e alta) a vulnerabilidade natural dos solos representativos das áreas de recarga do Aqüífero Guarani na região estudada.
\end{abstract}

PALAVRAS-CHAVE: VULNERABILIDADE, RISCO DE CONTAMINAÇÃO, AQÜÍFEROS, AGROQUÍMICOS

* Geólogo, Doctor Scientiae em Solos, Pesquisador da Embrapa Meio Ambiente, Jaguariúna, SP. (e-mail: gomes@cnpma.embrapa.br).

** Engenheiro Agrônomo, PhD. em Ciência de Solo-Água, Pesquisador da Embrapa Meio Ambiente.

*** Matemática, Doctor Scientiae em Matemática Aplicada, Pesquisadora da Embrapa Meio Ambiente. 


\section{INTRODUÇÃO}

O estudo do movimento de agroquímicos no perfil do solo envolve muitos fatores e isso tem contribuído para que o fenômeno seja ainda pouco conhecido no meio técnico-científico (TUON et al., 1995). Sabese, no entanto, que o movimento de agrotóxicos e nutrientes no solo ocorre por meio de difusão e pelo fluxo de massa em água (HUNTER e STABE, 1972), associado às transformações químicas e biológicas e processos de adsorção e persistência do produto (MERKLE et al., 1967; KANAZAWA, 1989; BARRIUSO et al., 1992).

O presente trabalho propõe um método simplificado de avaliação da vulnerabilidade natural do solo, como forma de subsidiar estudos relativos à movimentação de agroquímicos, sobretudo aqueles voltados à avaliação de riscos de contaminação da água subterrânea. Para tanto, considerou-se a relação entre condutividade hidráulica e declividade dos principais solos agrícolas brasileiros (Quadro 1), tomando por referência as classes de $1^{\circ}$, $2^{\circ}$ e $3^{\circ}$ níveis categóricos (EMBRAPA, 1999) para a obtenção de três classes gerais de vulnerabilidade, denominadas de baixa, média e alta. Entende-se vulnerabilidade como sendo a susceptibilidade de um compartimento, solo ou água, por exemplo, ser adversamente afetado por uma carga contaminante qualquer, conforme a proposição de FOSTER (1993) e do Instituto Geológico (SÃO PAULO, 1997) para aqüíferos.

\section{MATERIAL E MÉTODOS}

O presente estudo foi desenvolvido para os principais solos da microbacia do Córrego Espraiado, localizada no município de Ribeirão Preto/SP, mais precisamente entre as coordenadas $21^{\circ} 05^{\prime}$ e $21^{\circ} 20^{\prime}$ de latitude sul e $47^{\circ} 40^{\prime}$ e $47^{\circ} 50^{\prime}$ de longitude W.Gr. A altitude média é de $600 \mathrm{~m}$ e o relevo dominante do tipo suave ondulado. O solo é constituído predominantemente por Latossolo Vermelho Eutroférrico e Distroférrico, classificados pelo Sistema Brasileiro mais antigo como Latossolo Roxo dos tipos eutrófico e distrófico em proporções semelhantes (MIKLÓS e GOMES, 1996). Na porção mais jusante da área, representativa da recarga do Aqüífero Guarani, predominam solos arenosos do tipo Latossolo Vermelho Distrófico psamítico e Neossolo Quartzarênico (EMBRAPA, 1999).

O método adotado preconiza a influência de determinadas propriedades físicas do solo nos potenciais de infiltração e escoamento superficial da água. Propriedades como textura, estrutura, estabilidade 
de agregados e profundidade do solum (horizonte $A+B$ ), em conjunto, permitem estimar a condutividade hidráulica, aqui classificada em alta $(12,5$ a $25 \mathrm{~cm} / \mathrm{h})$, média $(2,5$ a $12,5 \mathrm{~cm} / \mathrm{h})$ e baixa $(0,25$ a $2,5 \mathrm{~cm} / \mathrm{h})$, conforme adaptação de KRAMER (1969) e exemplificada no Quadro 1.

\section{QUADRO 1 - CONDUTIVIDADE HIDRÁULICA ESTIMADA DOS PRINCIPAIS SOLOS AGRÍCOLAS BRASILEIROS, CONSIDERANDO AS CLASSES DE 1, 2 E $3^{\circ}$ NÍVEIS CATEGÓRICOS, EM FUNÇÃO DA TEXTURA, ESTRUTURA, ESTABILIDADE DE AGREGADOS E PROFUNDIDADE DO SOLO}

\begin{tabular}{|c|c|c|c|c|c|}
\hline Classe de Solo & $\begin{array}{c}\text { Textura } \\
\text { Dominante }\end{array}$ & $\begin{array}{c}\text { Estrutura } \\
\text { Dominante }\end{array}$ & $\begin{array}{c}\text { Estabilidade de } \\
\text { Agregados }\end{array}$ & $\begin{array}{c}\text { Profundidade } \\
\text { (Horiz. A+B) }\end{array}$ & $\begin{array}{l}\text { Condutivida } \\
\text { de HidrA凷ica }\end{array}$ \\
\hline \multirow{3}{*}{\begin{tabular}{|l|} 
Latossolos \\
Vermelhos \\
Eutroføricos, \\
DistrofØricos, \\
Aluminof $\varnothing$ ricos, \\
Eutr ficos e \\
Distr ficos \\
\end{tabular}} & Argilosa & $\begin{array}{l}\text { Blocos sub- } \\
\text { angulares }\end{array}$ & $\mathrm{Est} / \approx \mathrm{el}$ & Profundo & Alta \\
\hline & Argilo-Arenosa & Granular & Pouco Est/Ey el & Profundo & Mødia \\
\hline & Arenosa & Granular & Pouco est/4el & Profundo & Mødia \\
\hline \multirow{3}{*}{$\begin{array}{l}\text { Latossolos } \\
\text { Vermelho-Amarelos } \\
\text { Acriføricos, cricos, } \\
\text { DistrofØricos, } \\
\text { Distr ficos e } \\
\text { Eutr ficos }\end{array}$} & Argilosa & $\begin{array}{l}\text { Blocos sub- } \\
\text { angulares }\end{array}$ & $\mathrm{Est} /$ \& el & Profundo & Alta \\
\hline & Argilo-Arenosa & Granular & Pouco Est/的l & Profundo & MØdia/Alta \\
\hline & Arenosa & Granular & Inst/\&y el & Profundo & Alta \\
\hline \multirow{3}{*}{$\begin{array}{l}\text { Latossolos Amarelos } \\
\text { Acriføricos, cricos, } \\
\text { DistrofØricos, } \\
\text { Distr ficos e } \\
\text { Eutr ficos }\end{array}$} & Argilosa & $\begin{array}{l}\text { Blocos sub- } \\
\text { angulares }\end{array}$ & $\mathrm{Est} / \mathrm{E}_{\mathrm{el}}$ & Profundo & Mødia \\
\hline & Argilo-Arenosa & $\begin{array}{l}\text { Blocos sub- } \\
\text { angulares }\end{array}$ & Est/4rel & Profundo & MØdia \\
\hline & Arenosa & $\begin{array}{l}\text { Blocos sub- } \\
\text { angulares }\end{array}$ & $\mathrm{Est} / \mathrm{flel}_{\mathrm{el}}$ & Profundo & MØdia \\
\hline $\begin{array}{l}\text { Nitossolos } \\
\text { Vermelhos } \\
\text { DistrofØricos e } \\
\text { EutrofØricos }\end{array}$ & Argilosa & $\begin{array}{c}\text { Blocos } \\
\text { angulares }\end{array}$ & Est/tyel & $\begin{array}{l}\text { Relativamente } \\
\text { Pouco profundo }\end{array}$ & Alta \\
\hline \multirow[b]{2}{*}{$\begin{array}{l}\text { Argissolos } \\
\text { Vermelhos } \\
\text { Eutrof } \varnothing \text { ricos, } \\
\text { Distr ficos e } \\
\text { Eutr ficos }\end{array}$} & Argilosa & $\begin{array}{l}\text { Blocos sub- } \\
\text { angulares }\end{array}$ & Est/4rel & Pouco Profundo & MØdia \\
\hline & Argilo-Arenosa & $\begin{array}{l}\text { Blocos sub- } \\
\text { angulares }\end{array}$ & Est/的l & Pouco Profundo & MØdia \\
\hline \multirow{2}{*}{$\begin{array}{l}\text { Argissolos Vermelho- } \\
\text { Amarelos Eutr ficos, } \\
\text { Distr ficos, } \\
\text { Alum nicos } \\
\end{array}$} & Argilosa & $\begin{array}{l}\text { Blocos sub- } \\
\text { angulares }\end{array}$ & Est/4rel & Pouco Profundo & MØdia \\
\hline & Areno-argilosa & $\begin{array}{l}\text { Blocos sub- } \\
\text { angulares }\end{array}$ & Est $/$ trel & Pouco Profundo & Mødia/baixa \\
\hline \multirow{2}{*}{$\begin{array}{l}\text { Argissolos Amarelos } \\
\text { Distr ficos e } \\
\text { Eutr ficos }\end{array}$} & Argilosa & $\begin{array}{l}\text { Blocos sub- } \\
\text { angulares }\end{array}$ & Est/बrel & Pouco Profundo & Mødia/baixa \\
\hline & Areno-argilosa & $\begin{array}{l}\text { Blocos sub- } \\
\text { angulares }\end{array}$ & Est/fyel & Pouco Profundo & Mødia \\
\hline
\end{tabular}

Fonte: GOMES et al. (1996a); GOMES et al. (1996b). 
A classificação de condutividade hidráulica foi submetida a tratamento estatístico para validação do método envolvendo as variáveis textura, estrutura, estabilidade de agregados e profundidade do solo. Valores numéricos foram atribuídos para os níveis de cada variável, como por exemplo: textura argilosa $=2,0$; areno-argilosa $=1,0$ e arenosa $=0$. Com os dados definidos, numericamente, foi realizada análise fatorial discriminante (JUDEZ, 1988) para verificar se o parâmetro condutividade hidráulica poderia ser obtido apenas com as quatro variáveis citadas. $O$ método propõe as classes alta, média e baixa de condutividade hidráulica para cada classe de solo conforme sua proximidade ao centro de cada nível. A classificação de condutividade hidráulica, obtida pelas estimativas de densidades Kernel com amplitudes desiguais (SAS INSTITUTE, 1988), coincidiu com a classificação feita preliminarmente proporcionando consistência aos resultados obtidos (GOMES, et al. 1996a). Em seguida foram estabelecidas as classes de declividade do terreno, com adoção de três níveis principais e seus respectivos valores percentuais, conforme LEMOS e SANTOS (1982): a) baixa $(<3 \%)$; b) suave (3 a $8 \%$ ) e c) acentuada (8 a 20\%). Valores acima de $20 \%$ de declividade foram descartados nessa avaliação, pois referem-se a áreas com forte restrição agrícola.

A relação matricial entre as classes de condutividade hidráulica e de declividade resultou na classificação dos potenciais de infiltração e de escoamento superficial da água no solo (Quadros 2 e 3).

\section{QUADRO 2 - POTENCIAL DE INFILTRAÇÃO EM RELAÇÃO À DECLIVIDADE E CONDUTIVIDADE HIDRÁULICA DO SOLO}

\begin{tabular}{|l|c|c|c|}
\hline \multirow{2}{*}{$\begin{array}{l}\text { Condutividade } \\
\text { hidr/4tlica }\end{array}$} & \multicolumn{3}{|c|}{ Declividade do solo } \\
\cline { 2 - 4 } & Baixa $(<3 \%)$ & Suave (3 a 8\%) & Acentuado ( 8 a 20\%) \\
\hline Baixa & MØdio & Baixo & Baixo \\
\hline MØdia & Alto & Mødio & Baixo \\
\hline Alta & Alto & Alto & M Ødio \\
\hline
\end{tabular}

Fonte: GOMES et al., 1996a.

Os Quadros 2 e 3 evidenciam, em síntese, que os potenciais de infiltração e de escoamento da água mantêm relação inversa entre si.

Nesse estudo foi considerada a condição de lençol freático profundo a muito profundo para todos os solos, já que a zona saturada do Aqüífero Guarani encontra-se a cerca de 42 metros de profundidade 
na área estudada (a qual representa parte das chamadas " áreas de recarga" desse manancial subterrâneo).

\section{QUADRO 3 - POTENCIAL DE ESCOAMENTO SUPERFICIAL EM RELAÇÃO À DECLIVIDADE E CONDUTIVIDADE HIDRÁULICA DO SOLO}

\begin{tabular}{|l|c|c|c|}
\hline \multirow{2}{*}{$\begin{array}{l}\text { Condutividade } \\
\text { hidr/tetlica }\end{array}$} & \multicolumn{3}{|c|}{ Declividade do solo } \\
\cline { 2 - 4 } & Baixa $(<3 \%)$ & Suave ( 3 a 8\%) & Acentuado (8 a20\%) \\
\hline Baixa & MØdio & Alto & Alto \\
\hline MØdia & Baixo & MØdio & Alto \\
\hline Alta & Baixo & Baixo & MØdio \\
\hline
\end{tabular}

Fonte: GOMES et al., 1996a.

Potencial de infiltração classificado como alto (em decorrência de alta condutividade hidráulica e da baixa declividade da área) indica alta vulnerabilidade à contaminação do compartimento água do lençol freático, com possibilidade de chegada do produto contaminante até os corpos d'água mais profundos ou à zona saturada do aqüífero em discussão. Como o lençol freático nessa avaliação foi substituido pela zona saturada do Aqüífero, esse parâmetro passou a ser neutro e a ênfase transferida para a condutividade hidráulica do solo e a declividade do terreno.

Potencial de escoamento superficial classificado como alto, por sua vez, indica que a área é mais vulnerável à erosão e à contaminação dos corpos d'água superficiais. Haverá perda de fertilidade e remoção de pesticidas do compartimento solo, via transporte pluvial, até os cursos d'água.

\section{RESULTADOS E DISCUSSÃO}

A análise do Quadro 4, principalmente, em relação aos Latossolos Vermelhos Distróficos psamíticos e Neossolos Quartzarênicos (representativos das áreas de recarga do Aqüífero Guarani) mostra que esses solos exibem valores mais elevados de vulnerabilidade natural em relação aos demais. Isto significa que quando expostos a determinada carga potencialmente contaminante como agrotóxicos, por exemplo, podem oferecer algum risco de contaminação para o aqüífero ou para o lençol freático. 


\section{QUADRO 4 - CLASSIFICAÇÃO DA VULNERABILIDADE EM FUNÇÃODA INTEGRAÇÃO DE INFORMAÇÕES DE CONDUTIVIDADE HIDRÁULICA, DECLIVIDADE DO TERRENO, POTENCIAIS DE INFILTRAÇÃO, ESCOAMENTO SUPERFICIAL DA ÁGUA E PROFUNDIDADE DO LENÇOL FREÁTICO PARA OS SOLOS AGRICULTÁVEIS DA MICROBACIA DO CÓRREGO ESPRAIADO, MUNICÍPIO DE RIBEIRÃO PRETO (SP)}

\begin{tabular}{|c|c|c|c|c|c|c|}
\hline $\begin{array}{l}\text { Classe de } \\
\text { Solo }\end{array}$ & $\begin{array}{c}\text { Condutividade } \\
\text { Hidr } A \text { ⿶ lica }\end{array}$ & $\begin{array}{l}\text { Declivida- } \\
\text { de do } \\
\text { Terreno }\end{array}$ & $\begin{array}{c}\text { Potencial de } \\
\text { Infiltra a o de } \\
\text { gua }\end{array}$ & $\begin{array}{c}\text { Potencial de } \\
\text { Escoamento de } \\
\text { gua }\end{array}$ & $\begin{array}{l}\text { Profundi- } \\
\text { dade } \\
\text { Len ol } \\
\text { FreAfico* }\end{array}$ & $\begin{array}{l}\text { Vulnera- } \\
\text { bilidade }\end{array}$ \\
\hline $\begin{array}{l}\text { Latossolos } \\
\text { Vermelhos } \\
\text { EutrofØricos } \\
\text { e } \\
\text { Distrof Øricos } \\
\text { argiss licos }\end{array}$ & MØdia & Suave & MØdio & Mødio & $\begin{array}{c}\text { Profundo a } \\
\text { muito } \\
\text { profundo }\end{array}$ & Mødia \\
\hline $\begin{array}{l}\text { Nitossolos } \\
\text { Vermelhos } \\
\text { Eutroføricos } \\
\text { latoss licos }\end{array}$ & Mødia & Acentuada & Mødio & Mødio & Profundo & Mødia \\
\hline $\begin{array}{l}\text { Latossolos } \\
\text { Vermelhos } \\
\text { Distr ficos } \\
\text { psam ticos }\end{array}$ & Alta & Suave & Mødio/Alto & Mødio/Baixo & Profundo & $\begin{array}{l}\text { MØdia/ } \\
\text { alta }\end{array}$ \\
\hline $\begin{array}{l}\text { Latossolos } \\
\text { Vermelho- } \\
\text { Amarelo } \\
\text { Distr ficos } \\
\text { pl nticos }\end{array}$ & Mødia & Suave & MØdio & MØdio & Profundo & Mødia \\
\hline $\begin{array}{l}\text { Neossolo } \\
\text { QuartzarCáico }\end{array}$ & Alta & Suave & Alto & Baixo & $\begin{array}{c}\text { Profundo a } \\
\text { muito } \\
\text { profundo }\end{array}$ & Alta \\
\hline
\end{tabular}

* Considerado neutro pela referência exclusiva de profundo a muito profundo, representativa das áreas de recarga do Aqüífero Guarani na microbacia estudada.

Com os resultados contidos no Quadro 4, adaptado de GOMES et al., (1996b), foram elaborados os mapas de condutividade hidráulica (Figura 1) e de declividade (Figura 2) para os solos da microbacia do Córrego Espraiado. Combinando as informações obtidas (Figuras 1 e $2 \mathrm{e}$ Quadro 4) foi elaborado o mapa final, integrando os dados de potencial de infiltração, potencial de escoamento superficial e vulnerabilidade, conforme mostra a Figura 3.

A definição das classes de vulnerabilidade teve como principal referencial o potencial de lixiviação (Figura 3), já que a presente abordagem pretendeu subsidiar estudos de risco de contaminação de água subterrânea. Os valores de vulnerabilidade apresentados permitem orientação mais adequada para o gerenciamento ambiental de áreas agrícolas, sobretudo em relação à aplicação de agroquímicos. 
FIGURA 1 - CLASSIFICAÇÃO QUANTO A CONDUTIVIDADE HIDRÁULICA DOS SOLOS

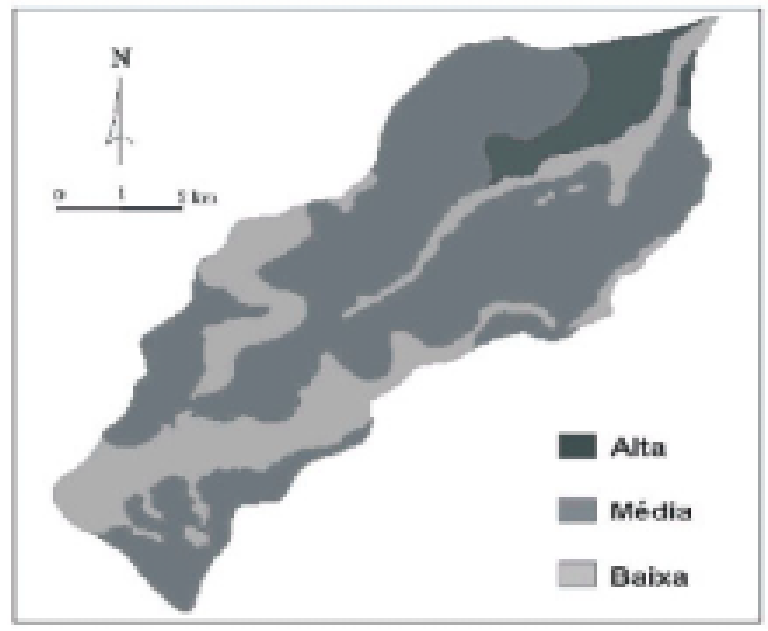

Fonte: GOMES et al. (1996b).

\section{FIGURA 2 - CLASSIFICAÇÃO QUANTO A DECLIVIDADE DO TERRENO}

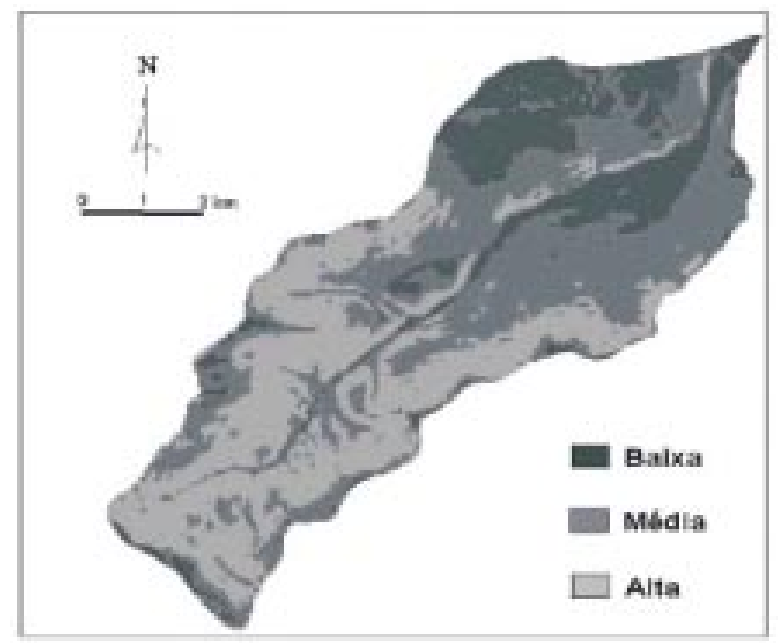

Fonte: GOMES et al. (1996b). 
FIGURA 3-POTENCIAL DE INFILTRAÇÃO POTENCIAL DE ESCOAMENTO DE ÁGUA NO SOLO E ESTIMATIVA DE VULNERABILIDADE - MICROBACIA DO CÓRREGO ESPRAIADO - RIBEIRÃO PRETO/SP

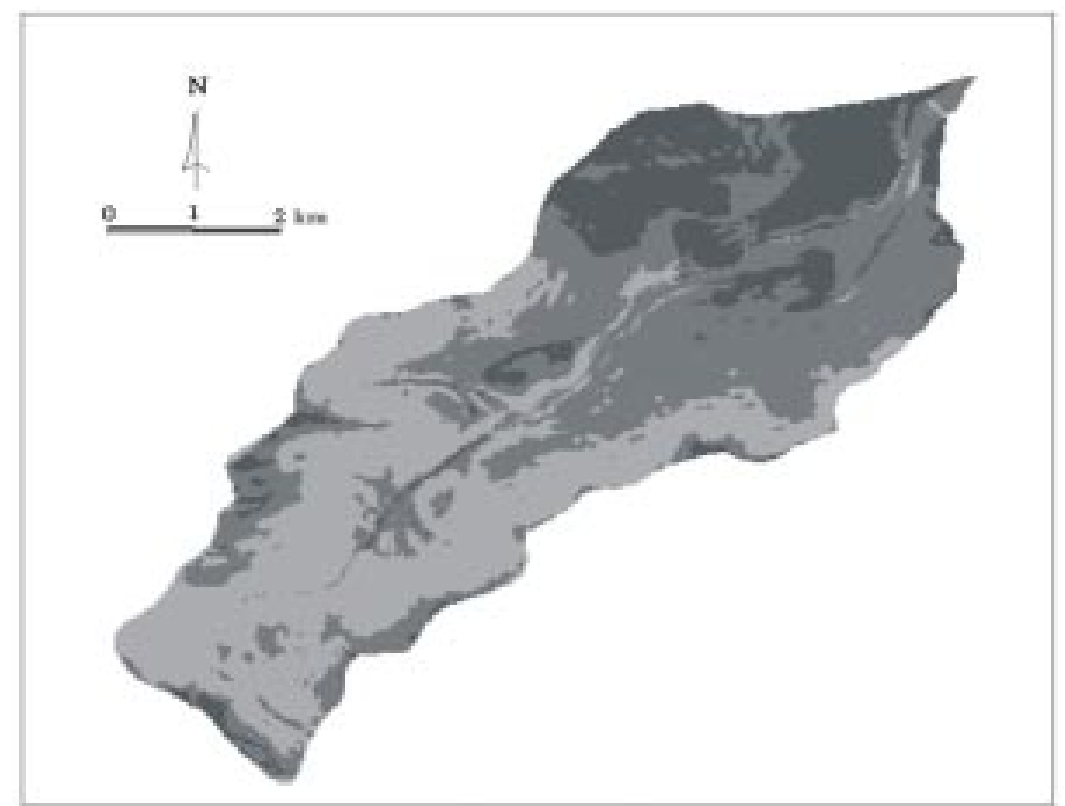

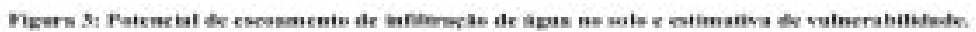

LEGNDA

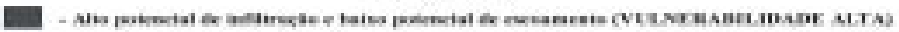

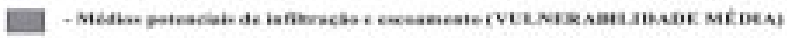

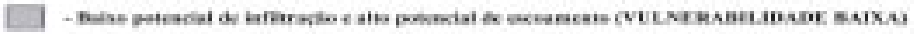

Fonte: GOMES et al. (1996b).

\section{CONCLUSÃO}

Concluiu-se que o potencial de infiltração de água no solo classificado como alto indica condição ambiental de vulnerabilidade alta do solo frente a carga contaminante, o que pode ser compreendido como condição favorável, a priori, à contaminação do lençol freático.

Para potencial de escoamento alto (vulnerabilidade baixa) o contaminante tende a se escoar superficialmente, seja em suspensão ou adsorvido a pequenos agregados ou torrões, tornando o solo menos exposto à condição de contaminação. Nesse caso, os cursos d'água 
tendem a ser comprometidos.

Os resultados apresentados permitem, ainda, orientar o gerenciamento rural no sentido de adoção de procedimentos que evitem riscos de contaminação do lençol freático. Entre esses procedimentos estão a seleção e aplicação de agrotóxicos que, preferencialmente, fiquem retidos no solo e que apresentem degradação rápida. Além dos agrotóxicos, os adubos nitrogenados devem ser selecionados, principalmente visando maior eficiência da planta na absorção de nitrogênio, uma vez que esse elemento nas suas diversas transformações no solo pode gerar compostos nocivos ao meio ambiente.

\section{Abstract \\ NATURAL VULNERABILITY OF SOIL IN AGRICULTURAL AREAS: SUBVENTION OF CONTAMINATION RISK ASSESSMENT OF GROUNDWATER BY AGROCHEMICALS}

The natural vulnerability of soil is a fundamental parameter in the studies of ambiental risk assessment, mainly in areas of great fragility as recharge areas of sedimentary aquifers. For this study it was adopted one typical area of direct recharge from Guarani aquifer, characterized by Espraiado watershed, localized in Ribeirão Preto region in São Paulo state, Brazil. The procedures consisted in the integration of the parameters hydraulic conductivity, declivity and depth of groundwater from soils of the area in study. The hydraulic conductivity was determined by the method of saturated column, with values considered from a estimation classified in low, medium and high. In this estimation, the integration of texture, structure, aggregate stability and soil depth data, which were submitted to statistical treatment for validation of the proposed method. The area declivity was obtained from planialtimetric maps with scale 1:10000, which allowed grouping in three classes: low, smooth and pronounced. Sequentially, it was established a matricial relation between the classes of hydraulic conductivity and declivity, resulting in the relative classification of infiltration potentials and superficial water flow for all watershed. It was concluded that this information combined to those relative to hydraulic conductivity, soil declivity and depth allowed, in a satisfactory way, to estimate in three classes (low, medium and high), the natural vulnerability of soils representatives of recharge areas of Guarani aquifer in the region studied.

KEY-WORDS: VULNERABILITY; CONTAMINATION RISK; AQUIFER; AGROCHEMICALS.

\section{REFERÊNCIAS}

1 BARRIUSO, E.; FELLER, C.; CALVET, R.; CERRI, C. Sorption of atrazine, tebutryn and 2,4-D herbicides in two Brasilian Oxisols. Geoderma, v. 53, p. 155-167, 1992.

2 DONZELLI, L.P. Levantamento aerofotogramétrico e georeferenciação de dados fisiográficos da microbacia do 
Córrego Espraiado em Ribeirão Preto-SP. Jaguariúna: EMBRAPA/CNPMA, 1996. 15 p. (Relatório final de consultoria).

3 EMBRAPA. Centro Nacional de Pesquisa de Solos. Sistema brasileiro de classificação de solos. Brasília: Embrapa Produção de Informação; Rio de Janeiro: Embrapa Solos, 1999. 412 p.

4 FOSTER, S. Determinação do risco de contaminação das águas subterrâneas: um método baseado em dados existentes. São Paulo: Instituto Biológico, 1993. 92 p.

5 GOMES, M.A.F.; SPADOTTO, C.A.; LUIZ, A.J.B.; NEVES, M. C. Método de classificação preliminar dos potenciais de infiltração e de escoamento superficial da água no solo: subsídio à avaliação do risco de contaminação por agroquímicos. In: CONGRESSO LATINO AMERICANO DE CIÊNCIA DO SOLO, 13., Águas de Lindóia, 1996. Anais... Águas de Lindóia: ESLAQ/SBCS, 1996a. 4 p. (Editado em CD ROM).

6 GOMES, M.A.F.; NEVES, M.C.; SPADOTTO, C.A.; LUIZ, A.J.B. Mapeamento expedito dos potenciais de infiltração e de escoamento superficial da água para os solos da microbacia do Córrego Espraiado em Ribeirão Preto-SP. In: CONGRESSO LATINO AMERICANO DE CIÊNCIA DO SOLO, 13., Águas de Lindóia, 1996. Anais... Águas de Lindóia: ESLAQ/SBCS, 1996b. 4 p. (Editado em CD ROM).

7 HUNTER, V.H.; STABBE, E.H. Moviment and persistence of picloran in soil. Weed Science, v. 20, p. 486-489, 1972.

8 JUDEZ, L. Técnicas de análisis de datos multidimensionales: bases teóricas y aplicaciones en agricultura. Madrid: Ministério de Agricultura Pesca y Alimentacion, 1988. $301 \mathrm{p}$.

9 KANAZAWA, J. Relationship between the soil sorption constants for pesticides and their pysicochemical properties.

Environ. Toxic. Chem., v. 8, p. 477-484, 1989.

10 KRAMER, P.J. Plant and soil water relationships: a modern synthesis. New York. McGraw-Hill Book Company, 1969. 482 p.

11 LEMOS, R.C.; SANTOS, R.D. Manual de descrição e coleta 
de solo no campo. Campinas: SBCS-SNLCS, 1982. 45 p.

12 MERKLE, M.G.; BOVERY, R.W.; DAVIS, F.S. Factors affecting the persistence of picloran in soil. Agron. Journal, v. 59, p. 413415, 1967.

13 MIKLÓS, A.A.W.; GOMES, M.A.F. Levantamento semidetalhado dos solos da Bacia Hidrográfica do Córrego Espraiado, Ribeirão Preto-SP. São Paulo, 1996. 48 p. (Relatório de Consultoria).

14 SÃO PAULO. Instituto Geológico. Mapeamento da vulnerabilidade e risco de poluição das águas subterrâneas no Estado de São Paulo. São Paulo: Instituto Geológico: CETESB, 1997. 2 v. (Série Documentos).

15 SAS INSTITUTE. SAS procedures guide. Cary, NC, 1988. 441 p.

16 TUON, R.L.; MONTEIRO, R.T.R.; LIBARDI, P.L.; COMPTE, V. Deslocamento miscível de $14 \mathrm{C}$ atrazina em colunas de solo. In: CONGRESSO BRASILEIRO DE CIÊNCIA DO SOLO, 25., Viçosa, 1995. Anais... Viçosa: UFV, 1995. p. 2404-2405. 